\title{
Securing Endotracheal Tube in Pediatric Neurosurgical Patients Operated in Prone Position-A Novel Technique
}

\author{
Rajkumar $^{1}$ Samir Kumar Kalraª \\ 1 UP University of Medical Sciences, Saifai, Etawah, Uttar Pradesh, India \\ ${ }^{2}$ Sir Ganga Ram Hospital \& Ganga Ram Institute of Medical \\ Education \& Research, New Delhi, India
}

Address for correspondence Rajkumar, MS, MCh, PhD, DSc FRCS, FAMS, FNS, FASET, MRCS, MNASc, UP University of Medical Sciences, Saifai, Etawah 206130, Uttar Pradesh, India (e-mail: vc@upums.ac.in, rajkumar1959@gmail.com).

Indian J Neurosurg 2021;10:241-243.

\begin{abstract}
Keywords

- endotracheal tube

- prone

- pediatric

Introduction The purpose of the present study relates to prevention of accidental extubation (AE) of pediatric patients in prone position. The control of endotracheal tube (ETT) in prone position becomes very difficult and an AE often occurs. This is more so deleterious in this age group because of the shallow respiratory reserves in children as well as the common use of noncuffed endotracheal tubes. The accidental tube exit may be a life-threatening event and its prevention is extremely important.

Case Description Using the used plastic bottle of intravenous fluids, a rectangular piece is cut, foam pasted and wrapped on itself and stout threads are attached. This attachment is used for securing the ETT by wrapping it. The technical details are described in the text. The AE is a potentially disastrous event and its occurrence in pediatric patients is even more dangerous. Many techniques have been developed over the years for prevention of such an event.

Conclusions The present technique uses all the principles of ETT fixation without leading to adding any significant technical difficulties. The method is safe, cost-effective and easily reproducible way of securement of ETT in pediatric neurosurgical patients operated in the prone position.
\end{abstract}

\section{Introduction}

Many of the neurosurgical procedures are performed in prone position and the control of the endotracheal tube (ETT) in this position becomes very difficult. There are numerous occasions when an accidental extubation (AE) takes place in this position. Such an AE is an emergency situation and demands immediate intervention, which would include change in position and sometimes mean removal of pin attachment securing the head, followed by turning the patient and reintubation as soon as possible. This could very easily be a life-threatening event, and in the past few years, it has been a focus of quality improvement programs related to endotracheal intubation.

AE may be potentially disastrous event and it is so due to a multitude of factors like repositioning of patient's head or

published online

May 13, 2021
DOI https://doi.org/

$10.1055 / \mathrm{s}-0040-1721204$

ISSN 2277-954X. the tube under drapes, decreased adhesiveness of plaster due to contact with the disinfectant or salivary secretions and weight of anesthesia circuit pulling on the ETT and the natural force of gravity in the prone position ${ }^{1,2} \mathrm{AE}$ not only leads to loss of airway but also other associated morbidities like tissue trauma to the face and airway, bronchospasm, aspiration pneumonia, and dysrhythmias., ${ }^{3,4}$

This can be encountered in any surgical procedure in the prone position and in any patient but assumes much more importance in the pediatric patients as the margin of safety is less due to smaller tube length and circumference, whether it is orotracheal or nasotracheal intubation. Moreover the tubes used are usually noncuffed in this age group. Better tube security is required in the pediatric patients and hence proper securing of the ETT is a must for safe conduct of pediatric neurosurgical procedure in the prone position. Nasotracheal intubation in 
pediatric patients is preferred in head and neck surgeries, as it provides better fixation and is less likely to kink $^{2-4}$ We propose a technique that reinforces the tracheal tube fixation, oral as well as nasal, leading to ETT securement, thus minimizing the chances of these kind of accidental extubations.

\section{Methods}

The technique makes use of a used plastic bottle (used for intravenous fluids) and a rectangular piece, which is cut approximately $5 \mathrm{~cm}$ in breadth and approximately 2 times long enough to wrap around the ETT to be used. A foam pad approximately1

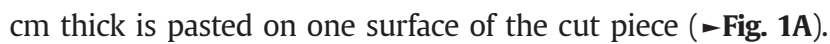
At one end of the piece, approximately $1 \mathrm{~cm}$ from the edge and in its middle and $2 \mathrm{~cm}$ above and below it, horizontal slit holes of size $0.5 \mathrm{~cm}$ are made. From the inner margin of these slit holes, the distance equal to the circumference of the ETT is measured across the piece and marked. In the same line as the three horizontal slit lines, from the part of the piece beyond the marked line, the cuts are made such that there remain three limbs approximately $1 \mathrm{~cm}$ broad centered on the horizontal slits ( - Fig. 1B). In the area between the cut slits and the three limbs, four horizontal slits of $0.5 \mathrm{~cm}$ each in two rows midway between the lines of three limbs are made ( - Fig. 1C). Then, as a next step, ribbon gauges approximately $1.5 \mathrm{~cm}$ in breadth are

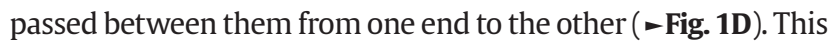
is the attachment ready for fixation to the ETT and has been named "Kumar and Kalra appliance" for ETT fixation. Following successful nasal intubation, the "Kumar and Kalra appliance" is wrapped around the ETT approximately $2 \mathrm{~cm}$ outside the entry into the nasal cavity and the three limbs are passed through the horizontal slits. This fits snuggly, since the outer diameter of
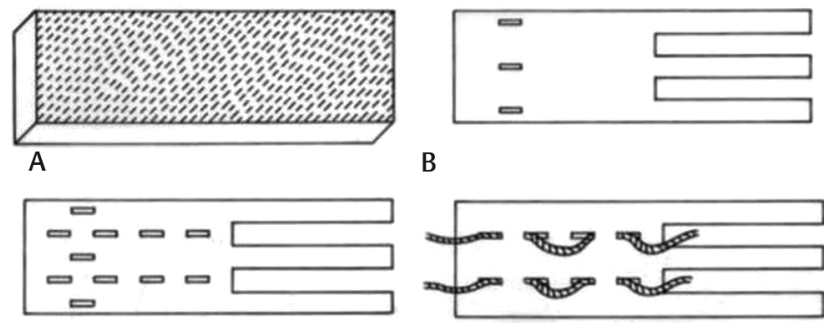

C
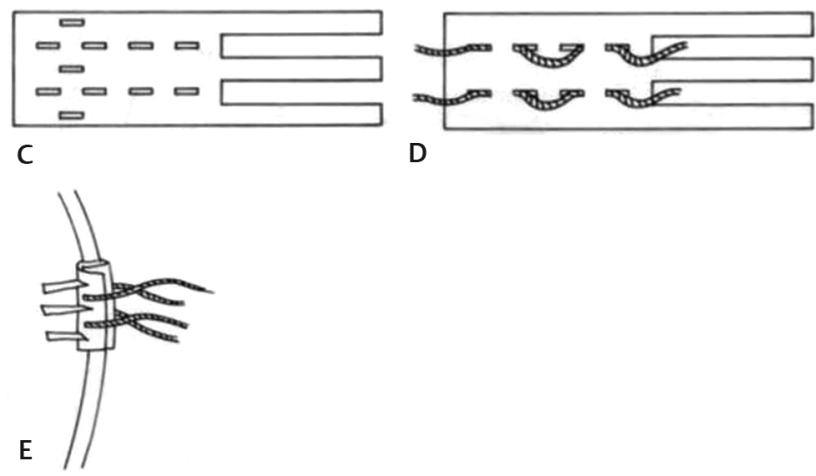

Fig. 1 Diagrammatic representation of (A) a plastic sheet, which is $5 \mathrm{~cm}$ broad and two times long enough to wrap around the endotracheal tube (ETT). Over the sheet, the wrapped foam pad $1 \mathrm{~cm}$ thick is shown. (B) The foamed sheet with three horizontal slits of $0.5 \mathrm{~cm}$ each $1 \mathrm{~cm}$ from one edge and three cut limbs of $1 \mathrm{~cm}$ breadth on the other end. (C) The four horizontal slits of $0.5 \mathrm{~cm}$ breadth in two rows. (D) The $1.5 \mathrm{~cm}$ broad ribbon gauges passed in the two rows of horizontal slits. (E) The "Kumar and Kalra attachment" wrapped around the endotracheal tube (ETT). the "Kumar and Kalra appliance" is identical to that of the ETT but the foam pad which is on the inside reduces the effective diameter of the "Kumar and Kalra appliance," and hence after tightening, hugs the tube firmly ( - Fig. 1E). The advantage of this wrapping is gravity helps in further fitting the attachment in a more snug manner. The limbs are locked on the horizontal slits by making vertical slits into them. The ribbon gauges are then tied across the back of head onto each other in cases of surgery outside the region of back of head and neck ( - Fig. 2). In the cases where the surgical field includes the back of head and neck, the two ends are tied to the end of the operation table. Thereafter, the rest of the fixation of the ETT with help of adhesive plaster over the face of the patient away from the mouth is performed as usual.

This technique was used at the Sanjay Gandhi Post Graduate Institute of Medical Sciences during the period January 2006 till June 2006 in 50 consecutive pediatric patients operated in prone position. The subset of patients operated were ( $\mathrm{M}: \mathrm{F}=27: 23$, age range: 6 months to 7 years) inclusive of encephalocele $(n=9)$, craniosynostosis $(n=7)$, craniovertebral junction anomalies ( $n=17$ ) and posterior fossa tumors ( $n=17$ ). The senior author continues to use the technique at his present institution, and this experience was of the time when the senior author was guiding the junior author at their common workplace.

\section{Discussion}

Reinforcements are usually needed and commonly used for pediatric patients to secure the ETTs, more so when they are operated in prone position. In neurosurgical practice, this requirement becomes more important because the surgical

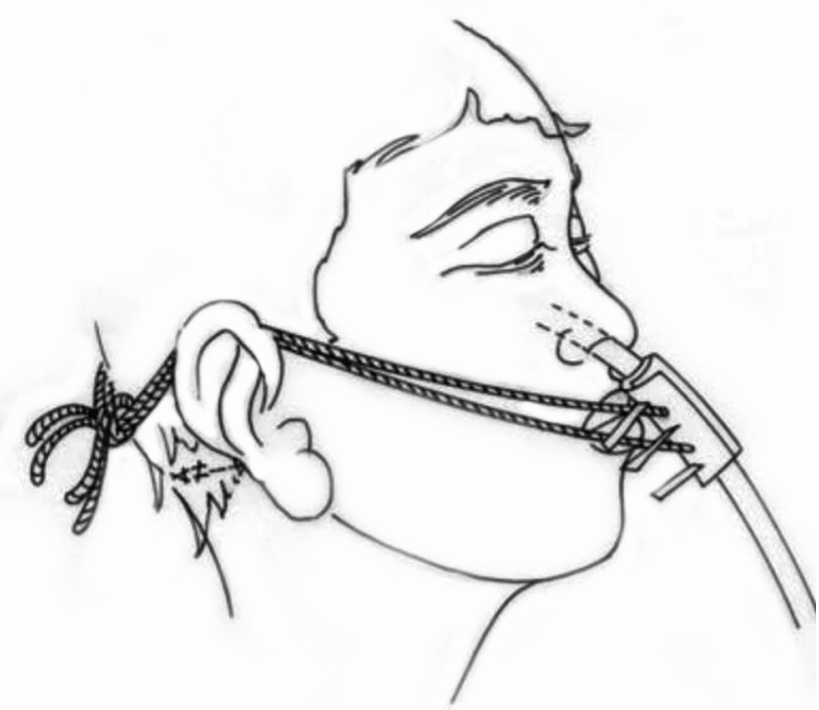

Fig. 2 Diagrammatic representation of the "Kumar and Kalra attachment" in place in the intubated patient, which is secured by wrapping around the head and neck. 
durations are long. The use of noncuffed tubes in this age group adds to this incidence, because the holding of the tube by cuff may also technically be of some help in preventing AE.

ETTS have to be secured effectively during performance of any surgical procedure where they are used. Any inadvertent extubation during surgery can be life-threatening, and the movement of the ETT can cause trauma to the laryngeal and tracheal mucosa ${ }^{1,5}$

AE has been realized as a potential disastrous event in patients who are operated in prone position. Many methods have been advocated to secure the nasotracheal tube ${ }^{1,2,5}$ The various methods of fixation of ETT in pediatric patients have been described, which include the use of stitching of the ETT through the nasal septum, using adhesive tapes alone, and along with adhesives such as tincture benzoin on the tube and skin, using transparent dressing all over the face to secure the ETT, and intranasal threading infant feeding tube to tie over ETT ${ }^{1,3}$ None of these methods have however been able to prevent the accidental slippage of ETT satisfactorily. Recently, a 4-component fixation of ETT has been described by Lovett et $\mathrm{al}^{4}$ which includes a) use of adhesive tape applied to the face and head in a variety of ways, b) cloth tape tied around the tube and around the neck and occiput, c) specialized devices using variety of hospital products, d) securement methods usually reserved for special situation like facial burns, fractures and oral and maxillofacial surgery.

The present technique in essence uses all the principles of ETT fixation, and it has been used during a period of 6 months in 50 consecutive pediatric neurosurgical patients operated in prone position. No accidental slippage of ETT was encountered during this time period. The repositioning of tube was also easy, since the use of plastic sheet as an attachment also provides the flexibility of molding along with the tube if needed. It was observed that the foam padding helped in snuggly fitting the attachment to the ETT and prevented any movement between the attachment and the ETT.

\section{Conclusions}

It is strongly felt that this is safe and cost-effective appliance. It is an easily reproducible method and should not take more that 5 to 7 minutes of time to make it. Its use can be extended to all patients who require ETT, since it does not incur any additional cost and hence can be used as a means of reinforcement of fixation of ETT. In nasotracheal intubations also, it can be easily secured in the same way. However, we need to use it in more patients to be able to come to a conclusion about its safety and efficacy. We also need to evaluate the efficacy of this technique in adult patients undergoing various types of surgical procedures.

\section{Acknowledgment}

The authors acknowledge the authorities of Uttar Pradesh University of Medical Sciences, Saifai, Etawah, Uttar Pradesh, for their approval and encouragement

\section{Conflict of Interest}

None declared.

\section{References}

1 Barnason S, Graham J, Wild MC, et al. Comparison of two endotracheal tube securement techniques on unplanned extubation, oral mucosa, and facial skin integrity. Heart Lung 1998;27(6):409-417

2 Clarke T, Evans S, Way P, Wulff M, Church J. A comparison of two methods of securing an endotracheal tube. Aust Crit Care 1998;11(2):45-50

3 Levy H, Griego L. A comparative study of oral endotracheal tube securing methods. Chest 1993;104(5):1537-1540

4 Lovett PB, Flaxman A, Stürmann KM, Bijur P. The insecure airway: a comparison of knots and commercial devices for securing endotracheal tubes. BMC Emerg Med 2006;6:7

5 Mikawa K, Maekawa N, Goto R, Yaku H, Obara H. Transparent dressing is useful for the secure fixation of the endotracheal tube. Anesthesiology 1991;75(6):1123-1124 